\title{
Alcohol Use History
}

National Cancer Institute

\section{Source}

National Cancer Institute. Alcohol Use History. NCI Thesaurus. Code C81229.

A description of an individual's current and past experience with alcoholic beverage

consumption. 